\title{
Carbon nanotubes in liquid crystals (Feature article)
}

\author{
Jan P. F. Lagerwall, ${ }^{a}$ and Giusy Scalia ${ }^{b, c}$ \\ Received (in XXX, XXX) 1st January 2007, Accepted 1st January 2007 \\ First published on the web 1st January 2007 \\ s DOI: 10.1039/b000000x
}

We review the research on carbon nanotube (CNT) dispersion in liquid crystals (LCs), focusing mainly on the approaches where the aim is to align CNTs along the LC director field, but covering briefly also the proposed possibility to enhance thermotropic LCs by CNT-doping. All relevant LC types are considered: thermotropic LC hosts allowing dynamic CNT realignment, lyotropic LC 10 hosts allowing very high concentration of CNTs uniformly aligned over macroscopic areas and consequent removal of the LC, and LC phases formed by CNTs themselves, used in spinning highquality carbon nanotube fibres. We also discuss the issue of CNT dispersion with some detail, since successful nanotube separation is imperative for success in this field regardless of the type of LC that is considered. We end by defining a few major challenges for the development of the field 15 over the next few years, critical for reaching the stage where industrially viable protocols for LCbased CNT alignment can be defined.

\section{Introduction}

What a typical carbon nanotube (CNT) and a typical liquid crystal (LC) researcher could perhaps have in common is that 20 they might not see any obvious connection between their respective research fields. While the two materials classes and their respective research communities-certainly are very different, a common treatment is actually much more rewarding than might be expected at first sight ${ }^{1}$, and they do 25 in fact share a few central features. Both involve highly anisotropic molecules and in both research fields long-range orientational order-resulting in macroscopic anisotropy-is of great importance. In fact, it is the combination of great contrasts and a few similarities that makes the meeting of ${ }_{30}$ CNTs and LCs so fruitful. It is the purpose of this feature article to explain this situation and to give examples of recent research where the promises of this new class of composite are well demonstrated.

Carbon nanotubes are exceptionally anisometric particles, 35 with diameters on the order of nanometers but lengths ranging from microns to centimetres ${ }^{2}$. Each CNT is like a graphene sheet rolled-up into tubular morphology or several such tubes arranged concentrically. The former is referred to as a singlewall carbon nanotube (SWCNT) whereas the latter generally 40 are lumped together in the broad class of multi-wall tubes (MWCNTs), although recent progress in synthesis has allowed the production of tubes with exactly two concentric carbon sheets, hence the introduction of the concept doublewall nanotubes (DWCNTs) ${ }^{3}$. The great interest in CNTs since 45 their discovery in the early 1990's is on the one hand due to the fascination over this new polymorph of carbon, on the other (and probably more important) to the catalogue of unique properties that it displays ${ }^{2}$. A perfect carbon nanotube is extremely resilient against mechanical stresses, the densityso normalized Young's modulus and tensile strength, respectively, of a typical SWCNT being some 20 and 60 times those of steel ${ }^{4}$. While real CNTs generally do not quite live up to these expectations-mainly due to defects-their actual mechanical properties are still truly outstanding, giving great 55 promise as fillers in novel light-weight but superstrong composites. A fundamental requirement is however that they can be well dispersed and preferably also aligned in the composite, and that the tubes interact strongly with the surrounding matrix.

${ }_{60}$ Of equal interest are the electronic properties of carbon nanotubes which, together with their inherent nanoscale wirelike shape, have given CNTs a proposed central role in future nanoscale electronic devices of a variety of kinds. An SWCNT can be metallic or semiconducting depending on its ${ }_{65}$ chirality, i.e. its particular spatial arrangement of the hexagonal carbon atom lattice in the tube, or 'how the graphene sheet is rolled up ${ }^{\dagger 2,5}$. Up until today, all synthesis methods of SWCNTs yield a random mixture of chiralities which means that, on the average, every third tube is metallic 70 whereas the others are semiconducting with a band gap on the order of $100 \mathrm{meV}$, the exact value depending on the diameter. Along the tube axis, a CNT has excellent electrical conductivity, reflecting the ballistic (scattering-free) transport of its nearly 1D electronic structure, allowing very high 75 currents with negligible heating. Should the nanotube nevertheless get heated (most likely by other processes than electrical conduction), the excess heat can also be very efficiently transported away: the room temperature thermal conductivity of an MWCNT has been measured ${ }^{4}$ to more than ${ }_{80} 3 \mathrm{~kW} / \mathrm{mK}$, greater than that of natural diamond and about eight times that of copper, giving carbon nanotube-based composites potential as excellent heat dissipaters.

All these attractive properties apply to the direction along the nanotube axis, the extreme anisometry of the CNTs giving ${ }_{85}$ them equally extreme anisotropic physical properties. As a consequence, control of nanotube orientation becomes a very important issue in most attempts to apply CNTs in devices or new materials, but this turned out to be challenging. Among the methods that have been proposed can be mentioned 90 magnetic and electric field alignment, shear flow or other 
mechanical techniques, as well as aligned growth. Field alignment certainly works, but magnetic fields of great strength are required (on the order of tens of $\mathrm{Tesla}^{6}$ ), whereas electric fields act differently on different CNTs due to their 5 varying electronic properties ${ }^{7}$. Furthermore, the alignment is in both cases maintained only as long as the field is applied, hence the tubes have to be fixed onto the target substrate or in the target matrix in the presence of the field. Attempts to align CNTs via shear flow have been discouraging, orientational 10 order parameters saturating well below $\mathrm{S}=0.1$ being reported ${ }^{8}$. Other mechanical techniques such as 'molecular combing, 9 are somewhat better but work only for very low CNT concentration and require specific treatments of the surfaces onto which the CNTs are deposited. The best of these 15 methods is probably aligned growth, where impressive progress has been reported the last few years, protocols for growth perpendicular ${ }^{10}$ as well as paralle $1^{11}$ to a substrate now having been developed. However, many synthesis methods are ruled out by the requirement to use a substrate and, more 20 important, the lack of chirality control and possibility to efficiently purify the product means that the tubes will have random electronic properties and be surrounded by impurities such as catalyst rests and amorphous carbon particles. Two different protocols for post-growth CNT fractionation 25 according to chirality and diameter have recently been described $^{12,13}$, but these require, just like most purification methods, non-supported nanotubes, free from any substrate. Finally, the aligned-growth methods are far from trivial and scale-up is challenging.

${ }_{30}$ This is where liquid crystals enter the picture. Although having much smaller molecular aspect ratio, LCs have the long-range orientational order that is such a challenge for CNTs inherently, the principal symmetry axis of the molecules (or molecule aggregates) spontaneously tending to 35 align along a common direction defined as the director (n). Moreover, the great response function to external forces (electric, magnetic, mechanical) of LCs in general gives us an easy means to define $\mathbf{n}$ as we wish over macroscopic areas, thermotropic liquid crystals even allowing the millisecond 40 dynamic reorientation of the director which is the basis for their use in modern display devices. Adding, finally, their fluidity, a requirement for usage as host for CNT dispersion, liquid crystals in many respects appear to be an ideal complement to unsupported carbon nanotubes. If the

45 nanotubes are well dispersed, they will in general align with their long axes along $\mathbf{n}$ in order to minimize distortions of the LC director field and thus the free energy, cf. Figure 1. Dispersion of CNTs in LCs can thus provide us with a cheap, simple, versatile and (as we will see below) effective means ${ }_{50}$ of controlling nanotube orientation on macroscopic scale and at high CNT concentration, with no fundamental restrictions on nanotube type. After having aligned the nanotubes, the LC may either be solidified (e.g. via polymerization) to produce a composite material with enhanced macroscopic mechanical, ${ }_{55}$ electronic and / or thermal properties, or it can be removed, a necessary step in the development of most CNT-based devices, which are generally incompatible with the LC matrix.

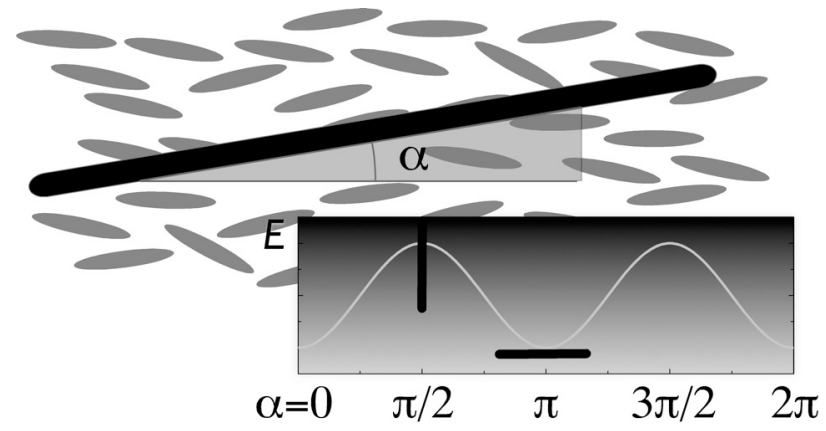

Figure 1. Schematic illustration of the CNT-in-LC alignment concept: the 60 system's free energy $E$ is minimized when the nanotubes align along the director $(\alpha=0, \pi, \ldots)$.

A number of reports have appeared recently suggesting a beneficial CNT-LC relationship also in the other direction, the LC behavior in electrooptic devices such as displays possibly ${ }_{65}$ being improved by the presence of small amounts of CNTs ${ }^{14-}$ 21. Although clearly an appealing concept, we will in this review only briefly touch upon this issue, as the observations are far from understood and have also been challenged by contradictory reports ${ }^{22,}{ }^{23}$. In contrast, the CNT-in-LC 70 alignment concept is by now well established with experimental studies by us and other groups clearly having demonstrated its potential ${ }^{24-31}$. Nevertheless, a number of important challenges still remain to be addressed before it can reach large-scale industrial usage. We will discuss both the 75 benefits and the difficulties in each type of CNT liquid crystal combination that may be of interest, including the case of liquid crystal phases formed by $\mathrm{CNTs}^{1,}{ }^{32-38}$, a highly interesting system that may form if one succeeds in dispersing a sufficiently high concentration of CNTs with good quality in 80 an isotropic solvent. In fact, the dispersion of CNTs is a fundamental challenge for any attempt to combine carbon nanotubes with liquid crystallinity, hence we will start the detailed discussion with this issue.

\section{A challenging but crucial requirement: efficient ${ }_{85}$ dispersion of CNTs}

Consisting entirely of carbon and having no edges ${ }^{\S 1}$, carbon nanotubes are chemically extremely inert and practically insoluble. Strong van der Waals interactions between adjacent nanotubes promote clustering into crystalline ropes which in 90 turn aggregate into strongly entangled and unorganized networks or bundles. Most solvents are useless in an attempt to prepare a suspension of carbon nanotubes, but a few exceptions have been identified, most notably dimethylformamide $(\mathrm{DMF})^{39}, \mathrm{~N}$-methyl-pyrrolidone $(\mathrm{NMP})^{40}$ and $\gamma$ ${ }_{95}$ butyrolactone $(\mathrm{GBL})^{41}$. The CNT-solvent interactions must here be unusually strong, comparable to the anti-dispersing CNT-CNT and solvent-solvent interactions, respectively ${ }^{40}$. Standard thermotropic liquid crystals such as 4-cyano-4' $-n$ pentylbiphenyl (5CB) also belong to the select category of 100 organic solvents that can disperse carbon nanotubes, and they are in fact surprisingly efficient at the initial stage ${ }^{42}$. Even gentle mechanical action is sufficient to get an immediate distribution of a CNT sample in a thermotropic nematic, a macroscopically homogeneous black suspension being the 
result already after just a few minutes of stirring. A more careful investigation however reveals that, while the LC is successful in separating macroscopic 'crumbs' of CNT into much smaller pieces, the nanotubes are still strongly bundled, 5 in aggregates large enough to be easily seen in an optical microscope. Moreover, the suspension is far from stable, most CNTs sedimenting on a time scale of days. Sonication can improve the situation somewhat, but until today, no combination of CNT and thermotropic LC has been identified 10 that allows long-term stable dispersions with high enough CNT concentration to give a macroscopically visible effect (darkening of the LC).

Several suggestions have been given for which types of interaction might be the relevant ones for CNT dispersion in 15 organic solvents, aromatic interactions arising as most likely being the dominant ${ }^{40}$. London-van der Waals (induced dipoleinduced dipole) and Debye-van der Waals (permanent dipoleinduced dipole) forces can be particularly strong due to the great polarisability along the tube axis of the extreme-aspect ${ }_{20}$ ratio CNTs, provided that the solvent molecule is either strongly polar (Debye) and / or highly polarisable (London). Obviously, this requirement is very well fulfilled by typical thermotropic mesogens, hence we can expect aromatic interactions to contribute to successful CNT dispersion in 25 thermotropic LCs. Indeed, a quantitative Raman study by Scalia et al. ${ }^{43}$ clearly showed that the aromatic and polar $5 \mathrm{CB}$ molecule interacts strongly with SWCNTs, as detected through a shift in the main peak of the CNT G-band (see below for a discussion of the CNT Raman response). Very 30 few studies ${ }^{43}, 44$ have so far dealt specifically with the questions related to CNT dispersion in thermotropic nematics, hence this is a topic open for future investigative efforts, theoretical contributions being particularly needed. The aims should on the one hand be to understand the success of 35 thermotropic nematics in achieving the initial coarse suspension, on the other to identify pathways to achieve single-tube dispersion at reasonable CNT concentration and stability.

In the carbon nanotube community the standard way to 40 disperse CNTs is not to use organic solvents, but rather to add them to a low-concentration (about $1 \mathrm{wt} .-\%$ ) aqueous surfactant solution, and submit this to high-power ultrasonication. The surfactant counteracts the van der Waals attraction of adjacent CNTs by introducing a sufficiently 45 strong repulsive force (electrostatic or steric) between them. A commonly used ionic surfactant is sodium dodecyl sulfate (SDS) but among the most efficient surfactants known are probably sodium dodecyl benzene sulfonate (SDBS) ${ }^{45}$ and bile salts like sodium cholate $(\mathrm{SC})^{46}$. Biological polyelectrolytes

${ }_{50}$ such as DNA have also turned out to be very useful for CNT dispersion $^{13,32,36,47}$. Nonionic surfactants are generally not quite as effective but decent results can be achieved with e.g. Triton X-100 48 .

At much higher concentration, the very same surfactant that ${ }_{55}$ is used for CNT dispersion will generally form lyotropic liquid crystal phases, where rod- or disc-shaped micelles are long-range ordered. However, as the amount of surfactant is increased in a CNT suspension, one must take the important phenomenon of depletion attraction into account ${ }^{49,50}$. When ${ }_{60}$ much more of a certain surfactant is present than what is necessary for covering the CNT surfaces, the surplus surfactant molecules organize into small empty micelles. Being repelled from the CNT-containing micelles, a system with high CNT concentration exhibits a large total excluded ${ }_{65}$ volume in which the small CNT-free micelles cannot penetrate. If two nearby CNT-containing micelles merge, they reduce the excluded volume considerably, hence an entropically driven attractive force between CNT-containing micelles appears. While this depletion attraction limits the 70 amount of CNTs that can be suspended, as well as the maximum concentration of surfactant, its exact role in liquid crystalline systems is somewhat unclear, as all modelling of depletion phenomena that we know of has been done for the case of spherical micelles forming an isotropic phase. The fact 75 that lyotropic LC phases have been successfully loaded with $\mathrm{CNTs}^{24-26,51}$ at a surfactant content well beyond the limit where an isotropic aqueous CNT-surfactant system was reported to suffer strongly from depletion attraction ${ }^{49}$ suggests that the special conditions of the LC phase has ${ }_{80}$ considerable impact on the phenomenon. In particular, one must probably take the impact of long-range orientational order of elongated micelles (both with and without nanotubes) on the total excluded volume into account. This is another area calling for further research efforts, experimental as well 85 as theoretical.

Before leaving the issue of dispersing CNTs we wish to stress that there is no such thing as a truly pure CNT sample. All SWCNTs and most MWCNTs are synthesized using catalysts, hence metal nanoparticles are likely to be present, 90 as are unwanted carbon particles such as graphite, amorphous carbon and non-tubular fullerenes. When interpreting the results of an experiment, one must thus be aware of the fact that, together with the CNTs, a fair amount of unwanted particles will have been added to the LC. In particular 95 researchers aiming to improve thermotropic LC properties by CNT addition must take great care to confirm without doubt that any observed effect is really due to the CNTs and not to impurities, but also when the aim is to verify CNT alignment, impurities may make the interpretation of data difficult.

\section{${ }_{100}$ CNTs in thermotropic liquid crystals}

The earliest attempts to employ the LC orientational order to align CNTs were all done using standard thermotropic nematic hosts such as $5 \mathrm{CB}$ or $\mathrm{E}^{27}, 30,31$. The advantages of thermotropic LCs in this context are twofold. First, using 105 existing alignment techniques, highly developed for the display industry, the ground state of the LC - and thus of the dispersed CNTs - can easily be determined on macroscopic scale. Second, the ease and speed of changing the director field by means of electric or magnetic fields adds a dynamic 110 quality to the system, in principle opening for switches based on reorientation of $\mathrm{CNTs}^{27},{ }^{28}$. On the other hand, thermotropics suffer from two severe problems, the first one being the already mentioned limited amount of CNTs that can be well dispersed with reasonable stability. This problem may 115 be overcome by materials development, optimizing the LC for 
CNT dispersion (to our knowledge, no single LC to date has been developed with this aspect in mind) or by functionalizing CNTs in a way that aids the dispersion in thermotropic LCs.

The second problem (for the cases where alignment is the ${ }_{5}$ purpose) refers to the fate of the LC after alignment has been achieved. While it in specific cases may be of interest to keep the CNTs in the host phase, for instance for dynamic devices or for composites formed via polymerization of the system, the vast majority of CNT applications are incompatible with 10 the LC phase, requiring a complete removal of the host without removing the CNTs or ruining the alignment that has been achieved. This is a truly challenging problem, in particular when using thermotropic LCs (as described below, lyotropic LCs may in this respect be more promising).

15 Vacuum suction of the LC through a porous membrane ${ }^{31}$ is to the best of our knowledge the only successful route that has been reported so far, but it is unclear how the CNTs could be transferred from the membrane to the target substrate.

The small limiting CNT concentration in thermotropics 20 also renders the proof of CNT alignment in this type of host a challenging task, the first reports providing only indirect data, from atomic force microscopy ${ }^{31}$ or from impedance spectroscopy ${ }^{27,}{ }^{30}$. In the first case the data did not reflect the actual CNT-in-LC suspension but instead what had been left 25 behind after LC with suspended CNTs deposited onto a porous membrane had been sucked through the pores. In the second, the interpretation of the data relied on the assumption that the conductivity increase with respect to the undoped LC was due strictly to the CNTs, but impurities typically present 30 in CNT samples could actually have a non-negligible influence on such measurements. With the employment of polarised Raman spectroscopy, unique in its ability to distinguish the nanotubes from the LC matrix and to detect extremely small quantities of CNTs due to the CNT Raman 35 resonance, a truly unambiguous verification of the LCinduced CNT alignment could finally be given using data collected from a CNT-LC composite in its natural liquid crystalline state ${ }^{29}$. SWCNTs have characteristic Raman modes in four regimes of the spectrum: the Radial Breathing Modes 40 (RBM), the Graphite (G) band, the Defect (D) band and, finally, the second-order response of the latter (sometimes called $\mathrm{G}^{\prime}$, sometimes $\mathrm{D}^{*}$ ). While the G-band is the strongest and therefore most informative when dealing with low CNT concentration or when the aim is quantitative analysis, the ${ }_{45} \mathrm{RBMs}$ are unique to SWCNTs, hence their presence unambiguously prooves that the signal is due to carbon nanotubes and not to other carbon species. Because nanotubes absorb light only if it is polarised along the tube axis and because the absorption of a photon is a necessary step in the

${ }_{50}$ Raman scattering process, the CNT Raman intensity is for all modes proportional to the degree of parallelism between the tubes and light polarisation ${ }^{52}$. This allows us to easily probe the CNT orientation by varying the polarisation of the excitation laser in the Raman set-up, cf. the example in Fig. 2.

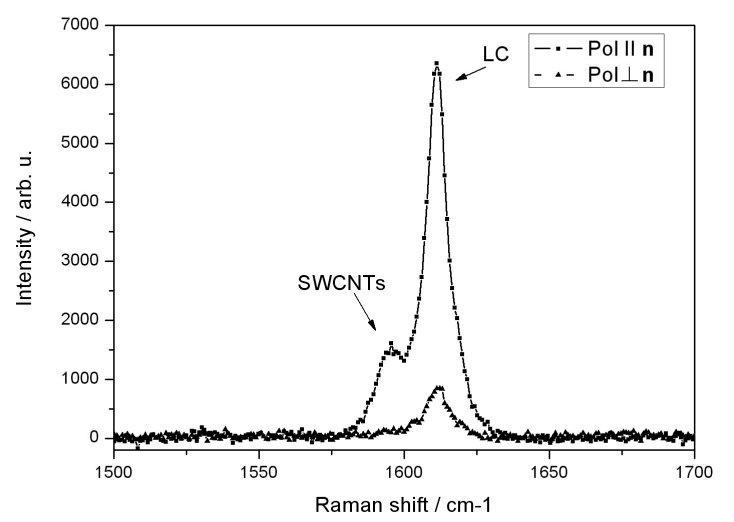

55

Figure 2. The G-band regime of Raman spectra from a HiPco SWCNT5CB LC composite taken with the polarisation of the excitation laser parallel and perpendicular to the director, respectively. The large polarisation dependence of the intensities of both peaks shows that the 60 CNTs and LC are aligned equally.

Recently, the polarised Raman technique was employed also to track the electric-field-induced switching of the LC in the vicinity of a SWCNT aggregate that was much too large to allow LC-alignment of the contained nanotubes ${ }^{21}$. In that case ${ }_{65}$ the CNTs influenced the director, rather than the other way around, stabilizing the starting configuration and counteracting the effect of the electric field. As a consequence, the switching threshold for the LC (the Frederiks threshold) was increased by a factor of about 4 . This 70 is a very important negative effect to keep in mind if one attempts to add CNTs to LCs for improving their performance in electrooptic devices.

Close to all work on composites of CNTs in thermotropic LCs has been done using nematic host phases, only two 75 exceptions being known to us. We investigated the effects of SWCNT doping on the mesomorphism of an antiferroelectric liquid crystal mixture exhibiting a particularly rich polysmectic phase sequence ${ }^{42}$, including the still mysterious intermediate chiral smectic-C phases ${ }^{53}$. Although the CNT 80 concentration in the LC was as small as $0.002 \mathrm{wt} .-\%$, their presence had a profound effect on the phase sequence, all chiral smectic-C-type phases except the ordinary $\mathrm{SmC}^{*}$ being suppressed. Whether the CNTs were aligned or not in the smectic host was not investigated as CNT alignment was not 85 the purpose of this study, but the fact that bundles were easily seen in the optical microscope suggests that only a minority of the CNTs can have been aligned. This may have contributed to the dramatic change in phase sequence, since disorganized bundles will disturb the smectic structure much more severely 90 than well-dispersed tubes. The other non-nematic study was done by Kumar and Bisoyi ${ }^{54}$, the host here being a hexagonal columnar phase formed by discotic mesogens, and the nanotubes being SWCNTs functionalized such that mesogenic triphenylene moieties were covalently attached outside the 95 tube walls. Covalent functionalization of CNTs has the drawback that it degrades the excellent properties of the CNTs (mainly the electronic), but it often yields much better solubility in a variety of hosts. Indeed, high loading of the 
functionalized CNTs was reported (up to 10\%) but unfortunately no assessment of dispersion quality was presented, nor of CNT alignment.

Several studies on LC-CNT composites have by now been ${ }_{5}$ published where the CNT orientation is not an issue. Instead, the focus is on the possible beneficial effects that the CNT guests may have on the LC host when it is used in an electrooptic device, e.g. a display. Reported effects include reduced effective ion content ${ }^{16,18,20,21}$, increased dielectric 10 anisotropy $^{16,17,19}$, suppressed backflow effects ${ }^{16,17}$, faster electrooptic response due to reduced rotational viscosity ${ }^{14}$ and lower switching threshold ${ }^{16,17,19}$. The CNT concentration was in all these works kept extremely low, yet substantial effects were reported. However, the effects have not always been 15 strictly beneficial for the display performance of the $\mathrm{LC}^{17,19}$, 22, 23: some studies report no improvement (often even a deterioration was observed), others report enhancement in some respects while degradation was observed in other parameters (which however were reported to be improved in 20 yet other studies).

On the whole, the situation is somewhat confused at present, partially due to a frequent lack of information on procedures and materials used for the experiments. Many of the reported effects-beneficial as well as adverse - might be 25 attributed to impurities such as amorphous carbon or metal particles, hence it is vital to assess the influence of these unwanted but unavoidable particles. The best way would probably be to conduct reference experiments where no CNTs but instead the impurity species are added to the LC, one at a

30 time and at controlled quantities. Only if the effects observed upon CNT doping are not seen in the reference experiments, can one be reasonably sure that they are indeed induced by the nanotubes. Finally, the explanations of the claimed effects are at present largely speculative, hence the advancement of this ${ }_{35}$ research field will critically depend on theoretical efforts.

\section{CNTs in lyotropic liquid crystals}

Although research on CNTs in lyotropic LCs is a few years younger than that based on thermotropic hosts, it is actually already further advanced in the senses that well separated ${ }_{40}$ CNTs at high concentration are possible and that schemes that can be used for advancing the research on CNT-based devices are now within reach. This situation is largely due to the fact that standard surfactants used for preparing high-quality CNT dispersions are perfect as the basis for making lyotropic LC-

${ }_{45}$ CNT composites. In contrast to the case of thermotropic LCCNT composites, one thus always starts by preparing an isotropic low-surfactant-concentration suspension of CNTs, and then this is made liquid crystalline by adding more surfactant ${ }^{24-26}$ or it is added to an already prepared lyotropic 50 liquid crystal sample ${ }^{51,55}$. Adding dry CNT powder directly to a preformed lyotropic liquid crystal phase works poorly ${ }^{55}$, as it is difficult to break up the large CNT aggregates of a dry CNT sample in such a viscous host.

In our initial work we used SDS for dispersing HiPco ${ }_{55}$ SWCNTs as well as for forming the LC phase ${ }^{25,26}$, the latter being a nematic phase forming at $25 \mathrm{wt} .-\%$ surfactant concentration if some $5 \mathrm{wt} .-\%$ of decanol is added as co- surfactant ${ }^{56}$. By tuning the exact composition of the final mixture we could select to have disc-shaped ${ }^{25}$ or rod-shaped ${ }^{26}$ 60 micelles forming the nematic phase. Up to a final CNT concentration of 0.01 wt.- $\%$ no CNT aggregation was observed upon inspection in a light microscope, so the CNTs were well dispersed, the bundle size being below optical resolution. As anticipated, the nanotubes were aligned along ${ }_{65}$ the director, for both micelle shapes, as verified by polarised Raman spectroscopy. The concept worked even upon addition of the chiral dopant tomatine rendering the host cholesteric (chiral nematic) with a pitch of its helical director modulation in the 100 micron range ${ }^{25}$.

70 Although the CNT loading with the SDS-based system was much higher than what is possible with bundle-free dispersion in thermotropic hosts, higher concentrations yet would be desirable. To achieve this, we modified our strategy slightly, switching to a better surfactant for the initial dispersion and 75 addressing the problem of depletion attraction at the same time. With SDBS some of the highest-concentrated SWCNT suspensions have been produced, single-tube dispersion at up to 2 wt.- $\%$ loading having been reported ${ }^{45}$, hence this surfactant allowed us to work with considerably more CNTs 80 in our suspensions. Rather than increasing the SDBS concentration to achieve liquid crystallinity, we now chose to use a different surfactant for producing the LC host phase. Specifically, we selected cetyl trimethyl ammonium bromide (CTAB) for this purpose, which at a concentration of 28 wt.${ }_{85} \%$ forms a hexagonal columnar lyotropic LC phase.

The great advantage of combining anionic SDBS for CNT dispersion with cationic CTAB for LC phase formation, is that we can achieve a complex of negatively charged nanotubecontaining dodecylbenzene sulfonate micelles surrounded by 90 positively charged empty cetyl trimethyl ammonium micelles. In contrast to the case where only a single surfactant is used for LC phase formation as well as for CNT dispersion, the empty micelles are now attracted to, rather than repelled from, the CNT-containing micelles, thereby not producing any ${ }_{95}$ depletion-induced aggregation of the nanotube guests. This type of electrostatic complexation has been reported for anionic DNA and cationic liposomes ${ }^{57}$ as well as for cationic poly(L-lysine) and lamellae of anionic lipids ${ }^{58}$, the driving force being the entropic gain by releasing the counter ions in 100 solution. Although the complexes in these studies always involve one polyelectrolytic species, we believe that the same mechanism is active in our system. The dodecyl benzene sulfonate molecules adsorb rather strongly to the CNT surface, most likely thanks to the aromatic interactions made ${ }_{105}$ possible by the presence of the phenyl ring between head group and tail, such that the surfactant-coated CNT effectively may behave like a polyelectrolyte. However, the long-range ordered hexagonal phase is a prerequisite for this to work: adding a dilute CTAB solution to the SDBS-dispersed CNTs 110 immediately leads to CNT aggregation. As the complexes are not stable in an isotropic phase, the result is instead a molecular-scale mixing of the two surfactants with CNT aggregation as a consequence. The complexation is in general rather sensitive to the experimental conditions under which 115 the system is prepared, and we are currently working at 
optimizing the process. In particular, a switch to DNA or another polyelectrolytic surfactant for dispersing the CNTs should render the system more robust.

A hexagonal columnar lyotropic host phase was also used 5 as host for SWCNTs by Weiss et al. ${ }^{51}$ and for MWCNTs by Jiang et al. ${ }^{55}$. While the latter team worked with a nonionic polyoxyethylene surfactant and an ionic liquid solvent for dispersing the CNTs as well as for forming the LC phase, the former worked with water-based systems, using the anionic 10 surfactant Triton X-100 for forming the LC host phase and the CNTs were dispersed either in Triton X-100 or in SDS. In both works, an enlarged spacing of the hexagonal columnar structure upon CNT introduction was detected by x-ray diffraction, and rheological measurements showed an

15 increased viscosity as CNTs were added to the LC. Based on tribological investigations of their system, Jiang et al. proposed the use of CNT-LC composites as high-performance lubricants. Unfortunately, the alignment of the CNTs in the host phase was verified in neither work.

${ }_{20}$ Compared to the single-surfactant system, the switch to the catanionic complex brought with it two substantial and very visible improvements. First, the concentration of CNTs was now so high (0.2 wt.-\%), and the alignment sufficiently good, that the CNT-LC composite acted as a linear polariser. As

25 already mentioned, CNTs absorb light polarised along the tube axis, hence an LC sample with a high enough concentration of well-dispersed CNTs aligned along $\mathbf{n}$ will absorb light polarised along $\mathbf{n}$, but not the perpendicular polarisation. The effect is really quite striking, because it

30 allows the CNT alignment to be verified directly by the naked eye: rotating a linear polariser held above a sample with the lyotropic CNT suspension illuminated from below with unpolarised light, the sample is distinctly darker when the polariser transmission direction is along the director than 35 when it is perpendicular to it, cf. Fig. 3. The anisotropic nanotube properties have been transferred to macroscopic scale by the liquid crystal. We should stress that a highquality dispersion of the CNTs is absolutely imperative for the effect to take place: in all cases where we have detected 40 bundling of the CNTs in the microscope, generally as a result of the initial isotropic CNT dispersion being of insufficient quality, no polariser effect whatsoever could be observed. Actually, even in the best cases, the contrast was not excellent, the bright state in Fig. 3 still being distinctly grey.

45 This reveals that the CNT orientational order was far from perfect on a macroscopic scale, but this is largely due to the imperfect macroscopic-scale control of the director orientation of the LC host. High-magnification Raman measurements revealed a higher degree of local order of the nanotubes ${ }^{24}$.

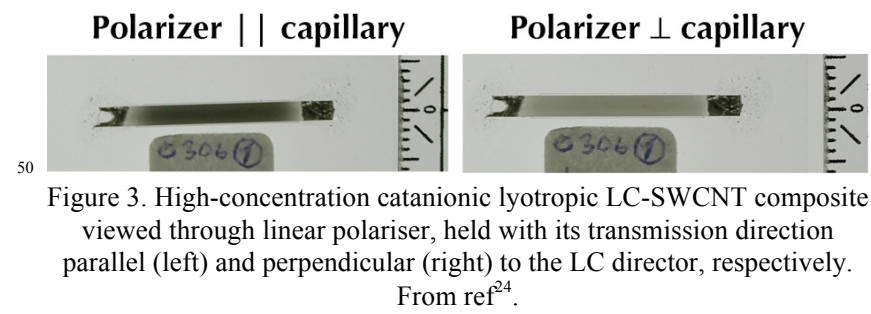

55 The second highly beneficial property of the new system is that thin filaments can very easily be pulled by simply dipping a sharp tip into a bulk sample and moving it gently away. In this filament, the director and consequently the SWCNTs are very well aligned longitudinally and, moreover, the filament ${ }_{60}$ can easily be transferred to a substrate, deposited along any desired direction or even with curvature, cf. Fig. 4a. By fixing the ends of the filaments to the substrate and then gently rinsing the sample with water, we could remove most of the surfactant. Raman experiments on the remaining CNTs 65 revealed that the nanotubes were very well aligned in the direction along which the filament had been deposited, cf. Fig. $4 \mathrm{~b}$, the orientational order parameter being about $S=0.7^{24}$. This observation is of great practical interest as it opens for a possibility to transfer the LC-aligned CNTs via the filaments 70 onto any desired target substrate. However, the rinsing step is far from trivial, our current procedure removing most, and in some cases all, of the CNTs. This step clearly requires a substantial research effort, sublimation of the surfactant possibly being an interesting alternative to rinsing. Once a 75 reliable procedure has been defined, this method can be employed for the development of a variety of CNT devices. In fact, as the lyotropic LC-alignment concept can easily be combined with both protocols for CNT fractionation according to chirality ${ }^{12,13}$ (an extension that we are currently 80 undertaking in our labs), it offers a unique opportunity for choosing not only CNT orientation but also its electronic properties, whether the desire is metallic CNTs or semiconducting tubes with a specific band gap. This is thus a very exciting area of development which may lead to a ${ }_{85}$ breakthrough in CNT device technology, once the remaining practical problem of efficient surfactant removal has been solved.

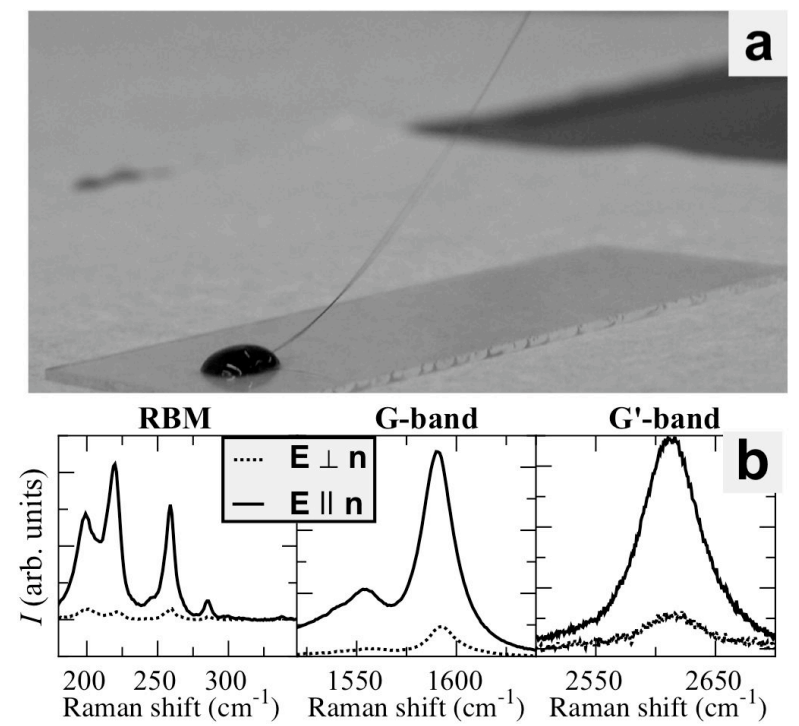

Figure 4. (a) Deposition of a filament of catanionic lyotropic LC-CNT 90 composite onto a glass substrate. (b; from ref. ${ }^{24}$ ) Raman spectra with polarisation parallel (fully drawn) and perpendicular (dotted) to the direction of filament deposition, after removal of surfactant by rinsing in water. 


\section{Liquid crystal phases formed by CNTs}

We end this review with a discussion of the case that the LC is not a host for the CNTs, but rather it is formed by the CNTs themselves, a research field that has been developed mainly by the groups around Windle ${ }^{34,35,38}$, Poulin ${ }^{32,36,49}$ and Smalley ${ }^{33,37,59}$. The basis for this phenomenon is the classical Onsager argument for liquid crystal phase formation in concentrated suspensions of rigid rods ${ }^{60,61}$ : if the aspect ratio of the rods and their concentration are both large enough, the 10 free energy of the system is reduced by forming a nematic phase, the cost of reduced orientational entropy being less than the gain in translational entropy (the latter corresponds to a decrease of excluded volume).

The Onsager theory predicts an onset of nematic order at a 15 volume fraction $v=3.3 d / L$, where $d$ is the diameter of the rods and $L$ is their length. With a typical SWCNT density of $1.5 \mathrm{~g} / \mathrm{mL}^{37}$ and a typical SWCNT $d / L$ ratio of $10^{-3}$ we would thus expect nematic phase formation already at about 0.5 wt.$\%$ loading. With MWCNTs some ten times higher loading 20 should be required. While experimental observations with MWCNTs $^{34,35,38}$ come close to the theoretical predictions, liquid crystallinity from SWCNTs have generally required higher loading than anticipated based on the Onsager theory ${ }^{36,}$ 37 , only one work ${ }^{32}$ coming close to the predicted $0.5 \mathrm{wt} .-\%$. ${ }_{25}$ Most likely, the main reason is the difficulty in completely separating the nanotubes from each other: even if the average SWCNT $d / L$ ratio may be on the order of $10^{-3}$, the aspect ratio of bundles can be substantially lower, in particular if the tubes are not uniformly aligned within the bundle. When successful 30 single-tube dispersion has been achieved, this has in almost all cases been at the cost of severe cutting of the tubes, a typical negative side effect of the high-power sonication required for breaking up the CNT aggregates. Finally, the Onsager theory has some limitations in that it assumes perfect 35 rod rigidity, which is not at all the case for long SWCNTs, and it considers only steric repulsive interactions. As already mentioned, strong attractive van der Waals forces are of great importance in CNT suspensions. When surfactants are introduced the situation is further complicated, not only by the 40 introduction of electrostatic repulsive forces, not accounted for by the theory, but also due to the effects of the surfactant sheath and the surrounding charged double layer on the effective rod geometry. On the one hand, the surfactant encapsulation increases the effective volume fraction

${ }_{45}$ considerably (at constant SWCNT weight fraction), on the other it does so at the expense of a great reduction in aspect ratio (a single surfactant molecule is typically twice as long as the SWCNT is thick).

The challenge in achieving liquid crystalline dispersion of ${ }_{50}$ CNTs lies in the dispersion: the method chosen must achieve high-quality CNT separation (preferably down to single-tube level) at high loading. Each of the three main groups active in the field has chosen its own approach. Whereas Windle and co-workers oxidized their MWCNTs to make them water ${ }_{55}$ soluble $^{34,35,38}$, the Smalley team dispersed pristine SWCNTs in super acids ${ }^{33}, 37,59$. The Poulin approach was instead to take help of $\mathrm{DNA}^{36}$ or hyaluronic acid ${ }^{32}$ for dispersing SWCNTs.
In all cases nematic LC phases were achieved as verified by birefringent textures detected in polarizing microscopy. ${ }_{60}$ Windle et al. also imaged disclinations in the nematic director field beautifully using scanning electron microscopy on samples from which the solvent had been evaporated ${ }^{34,} 38$. Obviously, the liquid crystalline structure remained intact upon drying.

${ }_{65}$ In terms of applicability, a drawback with the method of inducing LC phase behavior directly in CNT suspensions is that up until today, no method has been reported to control the director orientation, a prerequisite if one wants to use the method to deposit CNTs on a substrate along specific 70 directions. While uniform alignment under shear is possible, the macroscopic-scale order is lost when the shear stress is removed $^{62}$. If instead the aim is to achieve CNT fibers with a high degree of alignment, the shear-induced director control is however sufficient, as evidenced by the successful solution 75 spinning of carbon nanotube fibers from a nematic phase of SWCNTs suspended in super acid ${ }^{59}$.

Another interesting application of the LC phase formation of CNTs is fractionation according to length ${ }^{34}$. The Onsager theory was developed under the assumption of monodisperse 80 rods, an assumption that does not hold for a typical CNT sample. A treatment taking polydispersity into account reveals that if a sample is prepared with the right rod concentration for obtaining a biphasic isotropic + nematic sample, then long and straight rods will aggregate in the nematic regions, 85 whereas isotropic regimes will be enriched with short and / or curved tubes $^{34,61}$. After centrifuging such a sample, the LC phase will be collected at the bottom and the isotropic at the top, allowing a separation of the phases and rejection of the tubes in the isotropic regime. The procedure can be repeated, 90 each time narrowing down the dispersity in the LC phase that is kept, finally leading to a much more monodisperse CNT sample. While highly interesting on a lab scale and from a basic scientific point of view, the procedure might be too complex and time consuming to be applied industrially.

\section{${ }_{95}$ Conclusions and outlook}

We have in this article tried to show that carbon nanotubes in combination with liquid crystals form a highly interesting research area, with several potential industrially relevant applications in sight. While it has been shown without doubt 100 that CNT dispersion in LC phases can be very well used to align the CNTs, it is still an open issue if CNT doping can be used to enhance the performance of thermotropic LCs in displays or similar electrooptic devices. We can identify a few important main challenges which we believe will play a vital ${ }_{105}$ role for the continued development of the research field. For the case of CNT dispersion in thermotropic LCs, whether for nanotube alignment or for LC enhancement, synthesis efforts are called for, allowing high-quality dispersion of the CNTs at high concentration. While smart surfactant wrapping or 110 functionalization of the CNTs may improve dispersability in thermotropic LCs, this would most likely promote perpendicular anchoring of the LC molecules onto the CNT surface, a situation which creates great distortions of the LC director field and which does not provide a well-defined CNT 
orientation. LC mesogens capable of dispersing uncoated CNTs would thus be highly preferable. If high enough CNT concentration could be achieved without aggregation, the thermotropic LC-CNT composite could even allow a new type 5 of achromatic polarizer-free guest-host diplay ${ }^{24}$.

As for CNT dispersion in lyotropic LCs, high concentration together with good alignment has already been achieved and demonstrated, and the combination with fractionation procedures to achieve aligned as well as unichiral SWCNTs is

10 within reach with the first experiments under way. The main remaining challenge is the removal of the surfactant without removing the CNTs after deposition on the target substrate. While the only method proposed so far, using deposition of filaments of the LC phase, holds promise, the development of 15 alternative routes is still desirable.

The liquid crystal formation by CNTs themselves is also a highly interesting phenomenon which has already been used to spin fibers with well-aligned CNTs, and it can obviously provide important input for our basic understanding of LC ${ }_{20}$ phase formation of rod-like objects. As for the whole subject field of CNT research, an extended catalogue of efficient dispersion methods will be of great help also for the research in CNT-LC composites. Here experimental as well as theoretical efforts are called for, specifically addressing e.g. 25 the role of depletion attraction in LC phases or CNT-LC mesogen interaction mechanisms. On the other hand, other high-anisometry nanoparticles, e.g. inorganic nanotubes and nanowires ${ }^{63}$, may be easier to disperse, and also such particles should benefit from the orientational order offered by the LC

30 host. The future for LC-nanotube composites thus seems bright, with breakthroughs in carbon nanotube-liquid crystal composites probably being around the corner, and new research fields with other particles ready to be opened. We are confident that the research on this topic will produce some 35 very exciting results over the next few years.

\section{Acknowledgments}

Our work on CNT-LC composites would not have been possible without the constant support of S. Roth and ${ }_{40} \mathrm{~F}$. Giesselmann. Financial support from the Knut and Alice Wallenberg Foundation (JL) and the EU Marie Curie IntraEuropean Fellowship (GS) is gratefully acknowledged.

\section{Notes and references}

${ }^{a}$ Martin-Luther-Universität Halle-Wittenberg, Institut für Chemie, 45 Physikalische Chemie, Muehlpforte 1, 06108 Halle, Germany. Fax:+49 3455527 400; Tel: +49 3455525 836; E-mail: jan.lagerwall@chemie.uni-halle.de

${ }^{\mathrm{b}}$ Max-Planck-Institut für Festkörperforschung, Heisenbergstrasse 1, 70569 Stuttgart, Germany. Fax: XX XXXX XXXX; Tel: XX XXXX

50 XXXX; E-mail: g.scalia@ffkf.mpg.de

${ }^{\circ}$ ENEA Cr Portici, 80055 Portici (NA), Italy.

${ }^{\dagger}$ Of course, CNTs are not made by rolling up graphene sheets, but conceptually they can be very well visualized in this way.

${ }^{\S}$ Uncapped tubes have two edges, but they are negligible compared to the tube length.

1 C. Zakri, Liq. Cryst. Today, 2007, 16, 1 - 11.
2 R. Saito, M. S. Dresselhaus, G. Dresselhaus Physical properties of carbon nanotubes. Imperial College Press, UK, 1998,

3 J. L. Hutchison, N. A. Kiselev, E. P. Krinichnaya, A. V. Krestinin, R. O. Loutfy, A. P. Morawsky, V. E. Muradyan, E. D. Obraztsova, J. Sloan, S. V. Terekhov and D. N. Zakharov, Carbon, 2001,39, 761-770.

4 R. Baughman, A. Zakhidov and W. De Heer, Science, 2002, 297, 787-792.

5 A. Maiti, Nat. Mater., 2003, 2, 440-442.

6 J. E. Fischer, W. Zhou, J. Vavro, C. Llaguno, C. Guthy, R. Haggenmueller, M. J. Casavant, D. E. Walters and R. E. Smalley, J. Appl. Phys., 2003, 93, 2157-2163.

7 R. Krupke, F. Hennrich, H. von Lohneysen and M. M. Kappes, Science, 2003, 301, 344-347.

8 H. Wang, G. Christopherson, Z. Xu, L. Porcar, D. Ho, D. Fry and E. Hobbie, Chem. Phys. Lett., 2005, 416, 182-186.

9 S. Gerdes, T. Ondarcuhu, S. Cholet and C. Joachim, Europhys. Lett., 1999, 48, 292-298.

10 S. Maruyama, E. Einarsson, Y. Murakami and T. Edamura, Chem. Phys. Lett., 2005, 403, 320-323.; K. Hata, D. Futaba, K. Mizuno, T. Namai, M. Yumura and S. Iijima, Science, 2004, 306, 13621364.; S. M. Huang, L. M. Dai and A. W. H. Mau, J. Phys. Chem. B, 1999, 103, 4223-4227.

11 Y. G. Yao, Q. W. Li, J. Zhang, R. Liu, L. Y. Jiao, Y. T. Zhu and Z. F. Liu, Nat. Mater., 2007, 6, 283286.; A. Ismach and E. Joselevich, Nano. Lett., 2006, 6, 1706-1710.; S. Dittmer, J. Svensson and E. Campbell, Current Applied Physics, 2004, 4, 595-598.

12 M. Arnold, A. Green, J. Hulvat, S. Stupp and M. Hersam, Nat. Nanotechnol., 2006, 1, 60-65.

13 M. Zheng, A. Jagota, M. S. Strano, A. P. Santos, P. Barone, S. G. Chou, B. A. Diner, M. S. Dresselhaus, R. S. McLean, G. B. Onoa, G. G. Samsonidze, E. D. Semke, M. Usrey and D. J. Walls, Science, 2003, 302, 1545-1548.

14 H. Chen, W. Lee and N. Clark, Appl. Phys. Lett., 2007, 90, 033510.

15 S. Y. Jeon, S. H. Shin, S. J. Jeong, S. H. Lee, S. H. Jeong, Y. H. Lee, H. C. Choi and K. J. Kim, Appl. Phys. Lett., 2007, 90, 121901.

16 W. Lee, C. Wang and Y. Shih, Appl. Phys. Lett., 2004, 85, 513-515.

17 C. Huang, C. Hu, H. Pan and K. Lo, Jpn. J. Appl. Phys., 2005, 44, 8077-8081.

18 I. S. Baik, S. Y. Jeon, S. H. Lee, K. A. Park, S. H. Jeong, K. H. An and Y. H. Lee, Appl. Phys. Lett., 2005, 87, 263110.

19 C. Huang, H. Pan and C. Hsieh, Jpn. J. Appl. Phys., 2006, 45, 6392-6394.

20 H. Chen and W. Lee, Appl. Phys. Lett., 2006, 88, 222105 . 
21 G. Scalia, J. P. F. Lagerwall, S. Schymura, M. Haluska, F. Giesselman and S. Roth, Phys. Stat. Sol. (b), 2007, 244, 4212-4217.

22 C. Huang and H. Pan, Appl. Phys. Lett., 2006, 89, 056101.

23 S. Y. Jeon, S. H. Shin, J. H. Lee, S. H. Lee and Y.H. Lee, Jpn.J. Appl. Phys., 2007, 46, 7801-7802.

24 G. Scalia, C. von Buehler, C. Haegele, S. Roth, F. Giesselmann and J. P. F. Lagerwall, Soft Matter, 2008, 4, 570-576.

25 J. P. F. Lagerwall, G. Scalia, M. Haluska, U. Dettlaff-Weglikowska, S. Roth and F. Giesselmann, Adv. Mater., 2007, 19, 359-364.

26 J. P. F. Lagerwall, G. Scalia, M. Haluska, U. Dettlaff-Weglikowska, S. Roth and F. Giesselmann, Phys. Stat. Sol. (b), 2006, 243, 30463049.

27 I. Dierking, G. Scalia and P. Morales, J. Appl. Phys., 2005, 97, 044309.

28 I. Dierking and S. San, Appl. Phys. Lett., 2005, 87, 233507.

29 G. Scalia, M. Haluska, U. Dettlaff-Weglikowska, F. Giesselmann and S. Roth, AIP Conf. Proc., 2005, 786, 114.

30 I. Dierking, G. Scalia, P. Morales and D. Leclere, Adv. Mater., 2004, 16, 865-869.

31 M. Lynch and D. Patrick, Nano. Lett., 2002, 2, 1197-1201.

32 S. E. Moulton, M. Maugey, P. Poulin and G. G. Wallace, J. Am. Chem. Soc., 2007, 129, 9452-9457.

33 P. Rai, R. Pinnick, A. Parra-vasquez, V. Davis, H. Schmidt, R. Hauge, R. Smalley and M. Pasquali, J. Am. Chem. Soc., 2006, 128, 591-595.

34 S. Zhang, I. Kinloch and A. Windle, Nano. Lett., 2006, 6, 568-572.

35 W. Song and A. Windle, Macromolecules, 2005, 38, 6181-6188.

36 S. Badaire, C. Zakri, M. Maugey, a. Derre, J. Barisci, G. Wallace and P. Poulin, Adv. Mater., 2005, 17, 1673.

37 V. Davis, L. Ericson, A. Parra-vasquez, H. Fan, Y. Wang, V. Prieto, J. Longoria, S. Ramesh, R. Saini, C. Kittrell, W. Billups, W. Adams, R. Hauge, R. Smalley and M. Pasquali, Macromolecules, 2004, 37, 154-160.

38 W. Song, I. Kinloch and A. Windle, Science, 2003, 302, 1363-1363.

39 J. Liu, M. J. Casavant, M. Cox, D. A. Walters, P. Boul, W. Lu, A. J. Rimberg, K. A. Smith, D. T. Colbert and R. E. Smalley, Chem. Phys. Lett., 1999, 303, 125-129.

40 S. Giordani, S. Bergin, V. Nicolosi, S. Lebedkin, M. Kappes, W. Blau and J. Coleman, J. Phys. Chem. B, 2006, 110, 15708-15718.

41 S. D. Bergin, V. Nicolosi, S. Giordani, A. de Gromard, L. Carpenter, W. J. Blau and J. N. Coleman, Nanotechnology, 2007, 18, 455705.
J.P. F. Lagerwall, R. Dabrowski and G. Scalia, J. Non-Cryst. Solids, 2007, 353, 4411-4417.

43 G. Scalia, J. P. F. Lagerwall, M. Haluska, U. Dettlaff-Weglikowska, F. Giesselmann and S. Roth, Phys. Stat. Sol. (b), 2006, 243, 3238-3241. K. A. Park, S. M. Lee, S. H. Lee and Y. H. Lee, J. Phys. Chem. C, 2007, 111, 1620-1624. M. Islam, E. Rojas, D. Bergey, A. Johnson and A. Yodh, Nano. Lett., 2003, 3, 269-273. W. Wenseleers, I. I. Vlasov, E. Goovaerts, E. D. Obraztsova, A. S. Lobach and A. Bouwen, $A d v$. Funct. Mater., 2004, 14, 1105-1112.

M. Zheng, A. Jagota, E. D. Semke, B. A. Diner, R. S. Mclean, S. R. Lustig, R. E. Richardson and N. G. Tassi, Nat. Mater., 2003, 2, 338-342.; N. Nakashima, S. Okuzono, H. Murakami, T. Nakai and K. Yoshikawa, Chem. Lett., 2003, 32, 456457.; B. Gigliotti, B. Sakizzie, D. Bethune, R. Shelby and J. Cha, Nano. Lett., 2006, 6, 159-164.; H. Cathcart, S. Quinn, V. Nicolosi, J. M. Kelly, W. J. Blau and J. N. Coleman, J. Phys. Chem. C, 2007, 111, 66-74. C. Richard, F. Balavoine, P. Schultz, T. Ebbesen and C. Mioskowski, Science, 2003, 300, 775-778. C. Zakri and P. Poulin, J. Mater. Chem., 2006, 16, 4095-4098.

50 D. Marenduzzo, K. Finan and P. Cook, J. Cell Biol., 2006, 175, 681-686.

51 V. Weiss, R. Thiruvengadathan and O. Regev, Langmuir, 2006, 22, 854-856.

52 G. Duesberg, I. Loa, M. Burghard, K. Syassen and S. Roth, Phys. Rev. Lett., 2000, 85, 5436-5439.

53 J.P. F. Lagerwall and F. Giesselmann, Chemphyschem, 2006, 7, 20-45.

54 S. Kumar and H. Bisoyi, Angew. Chem. (Int. Ed.), 2007, 46, 1501-1503.

55 W. Q. Jiang, B. Yu, W. M. Liu and J. C. Hao, Langmuir, 2007, 23, 8549-8553.

56 L. Q. Amaral and M. E. Marcondes Helene, J. Phys. Chem., 1988, 92, 6094-6098.

57 C. R. Safinya, K. Ewert, A. Ahmad, H. M. Evans, U. Raviv, D. J. Needleman, A. J. Lin, N. L. Slack, C. George and C. E. Samuel, Philos Transact A Math Phys Eng Sci, 2006, 364, 2573-2596.; I. Koltover, T. Salditt, J. O. Radler and C. R. Safinya, Science, 1998, 281, 78-81.; J. Radler, I. Koltover, T. Salditt and C. Safinya, Science, 1997, 275, 810-814.

58 G. Forster, C. Schwieger, F. Faber, T. Weber and A. Blume, Eur Biophys J, 2007, 36, 425-435.

59 L. M. Ericson, H. Fan, H. Peng, V. A. Davis, W. Zhou, J. Sulpizio, Y. Wang, R. Booker, J. Vavro, C. Guthy, A. N. Parra-Vasquez, M. J. Kim, S. Ramesh, R. K. Saini, C. Kittrell, G. Lavin, H. Schmidt, W. W. Adams, W. E. Billups, M. Pasquali, W. F. Hwang, R. H. Hauge, J. E. Fischer and R. E. Smalley, Science, 2004, 305, 1447-1450. 60 1. Onsager, Ann Ny Acad Sci, 1949, 51, 627-659. 
61 A. Donald, A. Windle, S. Hanna. Liquid Crystalline Polymers. Cambridge University Press, Cambridge (UK), 2006,

62 P. Poulin, Personal Communication, 2008,

63 M. Remskar, A. Mrzel, Z. Skraba, A. Jesih, M. Ceh, J. Demsar, P. Stadelmann, F. Levy and D. Mihailovic, Science, 2001, 292, 479-481. 